\title{
Towards efficient near-infrared fluorescent organic light-emitting diodes
}

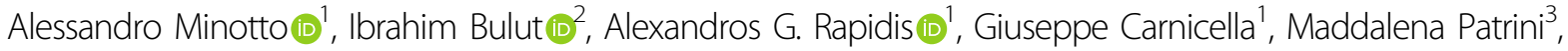 \\ Eugenio Lunedei (1) ${ }^{4}$, Harry L. Anderson ${ }^{2}$ and Franco Cacialli (iD)
}

\begin{abstract}
The energy gap law ( $E_{G}-$ law) and aggregation quenching are the main limitations to overcome in the design of nearinfrared (NIR) organic emitters. Here, we achieve unprecedented results by synergistically addressing both of these limitations. First, we propose porphyrin oligomers with increasing length to attenuate the effects of the $E_{\mathrm{G}}$-law by suppressing the non-radiative rate growth, and to increase the radiative rate via enhancement of the oscillator strength. Second, we design side chains to suppress aggregation quenching. We find that the logarithmic rate of variation in the non-radiative rate vs. $E_{\mathrm{G}}$ is suppressed by an order of magnitude with respect to previous studies, and we complement this breakthrough by demonstrating organic light-emitting diodes with an average external quantum efficiency of $\sim 1.1 \%$, which is very promising for a heavy-metal-free $850 \mathrm{~nm}$ emitter. We also present a novel quantitative model of the internal quantum efficiency for active layers supporting triplet-to-singlet conversion. These results provide a general strategy for designing high-luminance NIR emitters.
\end{abstract}

\section{Introduction}

Near-infrared (NIR) emitters are attracting significant interest for integration in a variety of applications, spanning from photodynamic therapy ${ }^{1}$ to security and defense ${ }^{2}$. Since NIR radiation (here defined as wavelengths $700 \mathrm{~nm}<\lambda<1000 \mathrm{~nm}$ ) is mostly invisible to the human eye, NIR light-emitting diodes (LEDs) are also among the best candidates for the development of Li-Fi (light-fidelity) all-optical networking systems, which offer a promising approach to the alleviation of bandwidth limitations often already affecting wireless (Wi-Fi) systems ${ }^{3,4}$. Furthermore, with respect to inorganic emitters, organic NIR emitters offer the possibility to further extend the range of applications thanks to their mechanical flexibility, conformability, and biocompatibility.

\footnotetext{
Correspondence: Harry L. Anderson (harry.anderson@chem.ox.ac.uk) or Franco Cacialli (f.cacialli@ucl.ac.uk)

'Department of Physics and Astronomy and London Centre for Nanotechnology, University College London, London WC1E 6BT, UK

${ }^{2}$ Department of Chemistry, Chemistry Research Laboratory, University of Oxford, Mansfield Road, Oxford OX1 3TA, UK

Full list of author information is available at the end of the article
}

Most of the recent research on NIR organic lightemitting diodes (OLEDs) ${ }^{5}$ has focused on rare-earth and transition metal complexes ${ }^{6,7}$, small molecules ${ }^{8,9}$, conjugated polymers, and their combinations ${ }^{9-14}$. However, the emission efficiency of organic emitters in the NIR is hindered by some intrinsic limitations. First, the extended conjugation length needed to achieve a sufficiently small energy gap $\left(E_{\mathrm{G}}\right)$ dictates a very planar molecular conformation, which in turn favors the formation of poorly emissive $\mathrm{H}$-type aggregates ${ }^{15}$. Undesired intermolecular interactions can be suppressed in conjugated systems by diluting the chromophores in solid solutions ${ }^{13,14,16-19}$ or via molecular design ${ }^{20}$, including threading into cyclodextrin rings to form conjugated polyrotaxanes ${ }^{21-23}$.

A potentially more significant hurdle is represented by the so-called "energy-gap law" ( $E_{\mathrm{G}}$-law) for radiationless transitions ${ }^{7,24}$, which predicts that the rate of the (nonradiative) transition between two electronic states increases exponentially as the energy difference $\left(E_{\mathrm{G}}\right)$ between the states is decreased. In fact, a concomitant decrease in the photoluminescence yield with $E_{\mathrm{G}}$ is commonly observed experimentally ${ }^{25}$, although it is

\section{(c) The Author(s) 2021}

(c) (i) Open Access This article is licensed under a Creative Commons Attribution 4.0 International License, which permits use, sharing, adaptation, distribution and reproduction cc) in any medium or format, as long as you give appropriate credit to the original author(s) and the source, provide a link to the Creative Commons license, and indicate if changes were made. The images or other third party material in this article are included in the article's Creative Commons license, unless indicated otherwise in a credit line to the material. If material is not included in the article's Creative Commons license and your intended use is not permitted by statutory regulation or exceeds the permitted use, you will need to obtain permission directly from the copyright holder. To view a copy of this license, visit http://creativecommons.org/licenses/by/4.0/. 
difficult, in general, to disentangle this contribution from that of aggregation.

Hybrid organic/inorganic innovative materials such as perovskite methylammonium lead halides ${ }^{26}$ and quantum $\operatorname{dots}^{27}$ may offer an alternative with high external quantum efficiency (EQE), but their heavy metal content will eventually prevent their use in most applications, especially biocompatible or wearable ones. Toxicity issues can also affect phosphorescent materials incorporating toxic heavy elements ${ }^{6,26,28-32}$.

Emission in the NIR by using heavy-metal-free materials has been achieved, for example, by leveraging triplets (e.g., via reverse intersystem crossing (RISC), or so-called "thermally activated delayed fluorescence (TADF)") but so far only at wavelengths $<800 \mathrm{~nm}$ for high efficiency devices $^{9,33,34}$ and, interestingly, also with fluorescent porphyrin oligomers ${ }^{18}$, whose conjugation length can be extended essentially across the entire molecule if the porphyrins are connected via conjugated bridges featuring one (meso-ethyne links ${ }^{35-37}$ ) or two triple bonds (mesobutadiyne links ${ }^{18,38}$ ). This scheme is particularly appealing for its potential tuneability, as by increasing the number of porphyrin units, the emission can be tuned from the visible range to wavelengths above $850 \mathrm{~nm}^{39}$, while still maintaining photoluminescence (PL) efficiencies of approximately $30 \%$ in dilute solution ${ }^{40}$.

\section{Results}

Here, we report the optical properties of a series of linear meso-butadiyne-linked zinc porphyrin oligomers (l-PN(THS) Fig. 1), and demonstrate unprecedentedly high EL EQEs by blending the zinc porphyrin hexamer $l$ P6(THS) in poly[(9,9-di- $n$-octylfluorenyl-2,7-diyl)-alt(benzo[2,1,3]thiadiazol-4,8-diyl)] (F8BT). The insertion of closed-shell zinc(II) metal ions into these porphyrin chains does not have a strong effect on their photophysical behavior, and the corresponding magnesium(II) complexes or free-base oligomers are expected to have similar properties. The choice of F8BT as a polymer host was driven mainly by its excellent semiconducting properties and energy transfer considerations; although we also note that the singlet exciton formation yield in F8BT OLEDs has been found to exceed the spin statistical limit of $25 \%$ in previous reports ${ }^{41,42}$.

In terms of emitter engineering, first, we propose a series of fluorescent heavy-metal-free porphyrin oligomers with increasing length (1-6 repeat units). Conjugated triple-bond-based bridges between the porphyrins allow effective intramolecular electronic coupling among the macrocycles, and thus enable the singlet exciton to delocalize over increasing portions of the molecule, thereby forcing an increasing mismatch of the spatial extent of the singlet and of the triplet excitons in view of the intrinsically localized nature of the triplets. Such a mismatch is expected to suppress intersystem crossing (ISC) and therefore the nonradiative rate $\left(k_{\mathrm{nr}}\right)$. In addition, exciton delocalisation is also expected to favour decoupling from vibrational ladders ${ }^{7}$. In fact, the growth of the nonradiative rate as a function of the decrease in $E_{\mathrm{G}}$ is characterized in our systems by a logarithmic rate approximately an order of magnitude smaller than in previous studies ${ }^{43-45}$. Moreover, the effective intramolecular electronic coupling among the porphyrins allows us to boost oscillator strength and thus radiative rate with increasing length. Second, we added trihexylsilyl (THS) side-chains to the aryl groups of the porphyrins to prevent aggregation quenching more effectively than in a previously reported linear hexamer ${ }^{18}$ thanks to steric hindrance by the THS groups, which limits $\pi-\pi$ interactions.

We are able to complement the above breakthrough by demonstrating EL peaked at a remarkably long wavelength of $850 \mathrm{~nm}$ from OLEDs with a polymeric active layer in which the hexamer is blended. Such OLEDs exhibited an average maximum EQE of $1.1 \%$, with values of up to $3.8 \%$ for the best performing devices. Such efficiencies are the highest reported so far for a heavy-metal-free NIR fluorescent emitter in this spectral region $(>800 \mathrm{~nm})$.

We also present a quantitative model that we have developed to describe the quantum efficiency of devices incorporating a guest-host emitter blend in which the host is susceptible to triplet-triplet annihilation (TTA) or TADF to afford further insight into device physics. The model supports the presence of TADF in addition to TTA as a mechanism to generate singlets via triplet conversion, whose existence is in turn supported by time-resolved EL data.

\section{Optical properties of I-PN(THS) oligomers in solution}

The absorption and PL spectra of the $l$-PN(THS) oligomers, measured in diluted toluene solution $\left(\sim 10^{-6} \mathrm{M}\right)$, are illustrated in Fig. 2.

The main spectral components in both the absorption and PL spectra are common to all the oligomers in the series and resemble those of similar non-THS-substituted oligomers $^{38-40}$. The high-energy absorption band ("Soretband") is due to transitions to the higher excited state $\left(S_{0}-S_{\mathrm{n}}\right)$ and peaks in the region $450-550 \mathrm{~nm}^{38}$, whereas the low-energy band ("Q-band", $S_{0}-S_{1}$ transition) is a manifold of species dominated by a low-energy component ${ }^{38}$. This component, named $Q_{\mathrm{X}}$, is strongly influenced by the number of porphyrin units in the oligomer.

As shown in Fig. 2a, the oscillator strength of the $Q_{x}$ transition increases with the number of porphyrin units due to the alignment of the corresponding dipole moment along the main axis of the oligomer, and the extended conjugation across the entire oligomer length ${ }^{46}$. For the same reasons, with increasing oligomer size, the $Q_{\mathrm{X}}$-band also gradually red-shifts from $630 \mathrm{~nm}$ ( $l$-P1(THS)) to 
a

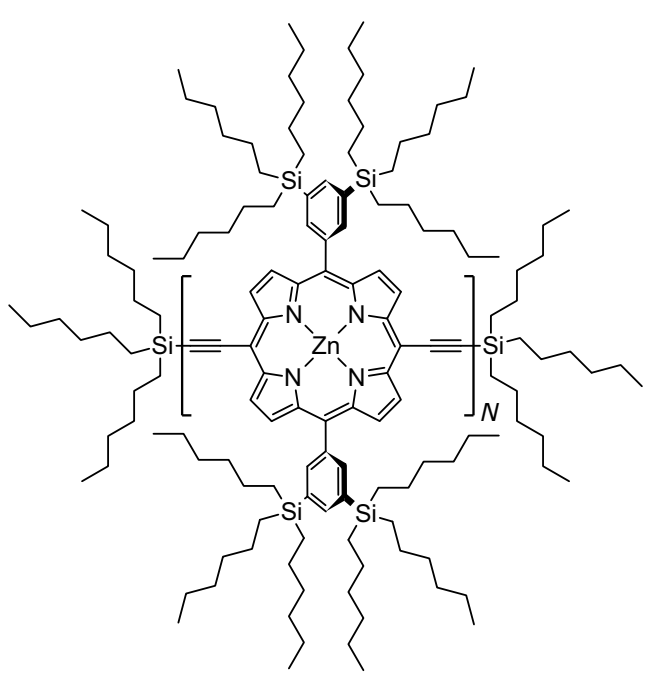

b

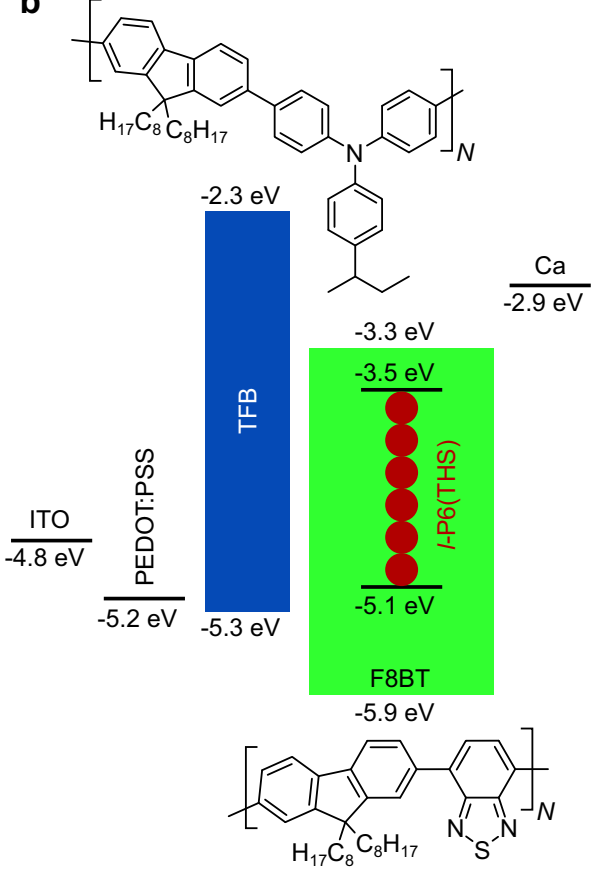

C

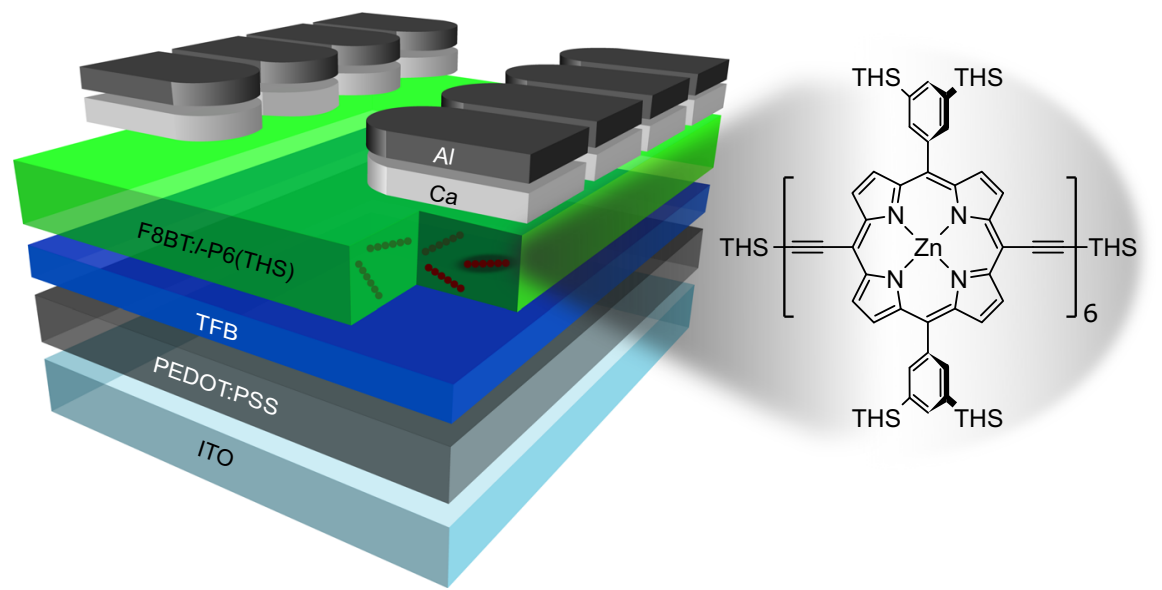

Fig. 1 Molecular structure of I-PN(THS) oligomers and OLED illustration. a Molecular structure of the I-PN(THS) oligomer series. $\mathbf{b}$ Band diagram for the materials employed in the OLEDs ${ }^{18,58}$. TFB (poly[(9,9-dioctylfluorenyl-2,7-diyl)-alt-(4,4'-(N-(4-sec-butylphenyl)diphenylamine)]) and F8BT molecular structures are illustrated above and below the relative band diagrams, respectively. c OLED architecture including an ITO patterned glass substrate, a poly(3,4-ethylene dioxythiophene) doped with poly(styrene sulfonate) (PEDOT:PSS) hole-transport layer, a TFB electron/exciton blocking layer, an F8BT:I-P6(THS) NIR light-emitting layer and a Ca/Al cathode

$770 \mathrm{~nm}$ ( $l$-P6(THS)). The overall broadening of the Q-band with increasing oligomer length can be ascribed to the torsional heterogeneity of the porphyrin units along the main axis, enabled by the meso-butadiyne links ${ }^{47,48}$.

In contrast to the ground state, in the $S_{1}$ excited state, from which fluorescence arises, the torsional disorder is reduced thanks to the much steeper potential energy surface, which favors a planar conformation. For this reason, twisted conformers that are excited from $S_{0}$ to $S_{1}$ tend to planarize prior to emission (on the $\sim 100 \mathrm{ps}$ timescale $)^{39,47,48}$. As a consequence, the fluorescence spectra in solution (Fig. 2b) exhibit a relatively narrow (70-130 meV full width at half maximum, FWHM) peak, which shifts from $630 \mathrm{~nm}$ to $800 \mathrm{~nm}$ with the number of porphyrins, and secondary vibronic components in the lower energy tail of the spectrum. An important property for NIR applications is that the fraction of photons emitted in the NIR $(>700 \mathrm{~nm})$ increases from $13 \%(l$-P1 (THS)) for the monomer to $>90 \%$ for the dimer, reaching $100 \%$ for the hexamer. 

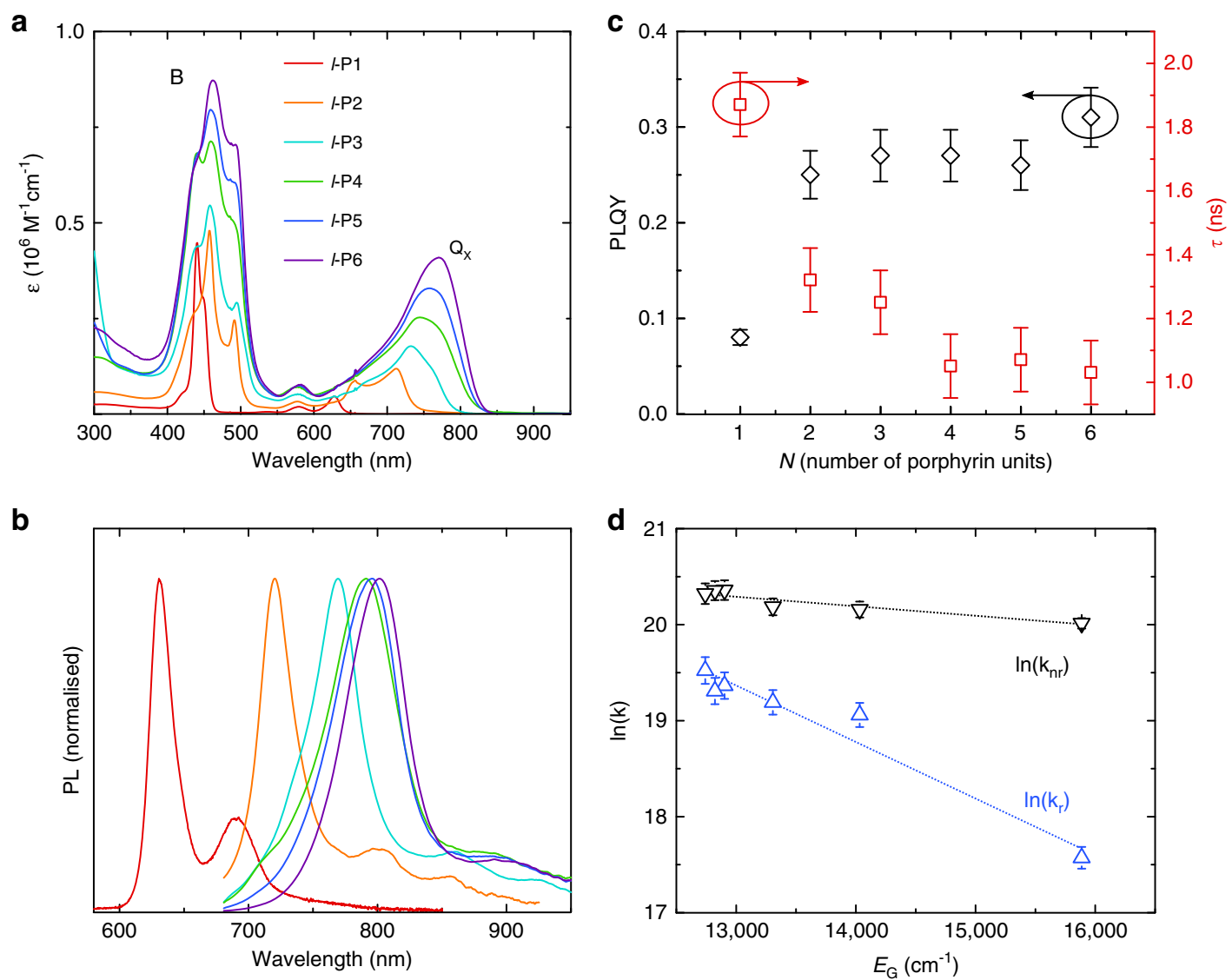

Fig. 2 Solution optical properties of I-PN(THS) oligomers. a Molar absorption coefficient $(\varepsilon)$ of the I-PN(THS) oligomer series in toluene solution. b PL spectra of the I-PN(THS) oligomers in toluene solution $(\sim 1 \mu \mathrm{M})$ measured at room temperature following excitation at $450 \mathrm{~nm}$. c Experimental PL quantum yield (PLQY) (black diamonds) and PL lifetime (red squares) versus oligomer length measured in $\sim 1 \mu \mathrm{M}$ toluene solutions at room temperature following excitation at $450 \mathrm{~nm}$ (PLQY) and $405 \mathrm{~nm}$ (PL lifetime). d Natural logarithm of radiative ( $k_{\mathrm{r}}$, blue triangles) and non-radiative ( $k_{\mathrm{nr}}$, black triangles) rates versus energy gap $\left(E_{\mathrm{G}}\right)$. The values of $k_{\mathrm{r}}$ and $k_{\mathrm{nr}}$ (in $\mathrm{s}^{-1}$ units) were derived from the experimental PLQY and PL lifetimes. The absolute value of the slope of $\ln \left(k_{n r}\right)$ is $\sim 0.86 \pm 0.16 \mathrm{eV}^{-1}$, i.e., more than one order of magnitude lower than those obtained from different series of fluorescent and phosphorescent dyes in previous studies $\left(\sim 10 \mathrm{eV}^{-1}\right)^{43-45}$. Dotted lines represent linear fits of the $\ln \left(k_{\mathrm{r}}\right)$ and $\ln \left(k_{\mathrm{nr}}\right)$ data. The linear fit of $\ln \left(k_{\mathrm{r}}\right)$ is presented only as a guide to the eye to highlight the different trend with $N$ (decreasing $\left.E_{\mathrm{G}}\right)$ compared to $\ln \left(k_{\mathrm{nr}}\right)$.

In Fig. 2c, we present the PL efficiency values for the $l$-PN(THS) series in toluene solution and the corresponding PL lifetimes $(\tau)$, which we obtained by fitting the mono-exponential PL decay of the same solutions (see Fig. S1, Supplementary Information). As expected, the lifetimes are $\sim 1-2 \mathrm{~ns}$, confirming the singlet nature of the radiative exciton, and decrease monotonically from $\sim 1.9 \mathrm{~ns}$ for $l$-P1 to $\sim 1.0 \mathrm{~ns}$ for $N>3$. This decrease is due to the increase in both the radiative $\left(k_{\mathrm{r}}\right)$ and non-radiative rates $\left(k_{\mathrm{nr}}\right)$ with oligomer length, as discussed below.

Most notably, however, as shown in Fig. 2c, the PLQY for $N>2$ essentially saturates at $\sim 28 \pm 3 \%$, more than three times higher than the PLQY of the monomer (8\%); this is rather surprising considering the decrease in the energy gap, i.e., this result is in apparent contrast with the predictions of the $E_{\mathrm{G}}$ law.
In fact, from the trends in the experimentally determined rates (Fig. 2d) extracted by combining the experimental PLQY and $\tau$ (Fig. 2c), we see that $k_{\mathrm{nr}}$ is not increasing nearly as dramatically as would have been expected on the basis of previous experiments ${ }^{43-45}$, contrary to the expectation of efficient internal conversion from $S_{1}$ to $S_{0}$. We propose that the increase in $k_{\mathrm{nr}}$ is prevented by the concomitant suppression of ISC, which is expected to decrease significantly with increasing oligomer length, mainly as a result of the increasing difference in spatial extent between singlets and triplets ${ }^{46,49,50}$, and possibly by suppression of exciton-vibrations coupling ${ }^{7}$.

Figure $2 \mathrm{~d}$ provides additional insight into the advantage of our material design strategy in affording a high PLQY by the simultaneous manipulation of both radiative and 
non-radiative processes. Specifically, we highlight the dependence of $k_{\mathrm{r}}$ on $E_{\mathrm{G}}$ as a consequence of the increasing oscillator strength with $N$.

Overall, both the enhancement of $k_{\mathrm{r}}$ and the suppression of ISC/excitonic-vibrational coupling in $l$-PN(THS) oligomers result in an exception to the corollary of the $E_{\mathrm{G}}$-law, i.e., that the PLQY should decrease with decreasing $E_{\mathrm{G}}$. This is very encouraging in the context of NIR emitters, as it allows us to develop devices with unprecedented EQEs by incorporating them into appropriate host matrices.

\section{Optical properties of $I$-P6(THS) in polymer matrices}

To take advantage of these promising properties and prevent aggregation quenching in solid-state devices, the next hurdle is the identification of a host featuring good charge transport and optical properties, especially in regard to spectral overlap and thus resonant energy transfer. Here, we selected the polyfluorene derivatives F8BT and poly(9,9-dioctylfluorene-alt- $\mathrm{N}$-(4-sec-butylphenyl)-diphenylamine) (TFB), which gave the best results (F8BT) for a number of low-gap emitters in previous work $^{14,18,19}$, or should have yielded good spectral overlap (TFB) between the host emission and the Soret bands of the porphyrins (the strongest ones, Fig. 2). As an emitter, we chose the hexamer among all oligomers because of its highest PLQY and NIR spectral purity.

The absorption and PL spectra for pure F8BT and blends with $l$-P6(THS) are reported in Fig. 3. The results for the TFB blends, which, despite the better spectral overlap, yielded devices with poorer performance, are reported in Figs. S3 and S4 and Tables S2 and S3 (Supplementary Information). We attribute the better performance of F8BT-based devices to better transport properties and higher singlet exciton formation rates (i.e., leveraging triplet-triplet annihilation or thermally activated delayed fluorescence, vide infra $)^{41,42}$.

As illustrated in Fig. 3a, the UV/Vis part of the blend film absorption spectrum is dominated by the F8BT components at 325 and $465 \mathrm{~nm}$, whereas the $Q_{\mathrm{x}}$ band of l-P6(THS) rises in the $800-900 \mathrm{~nm}$ range, with a maximum varying from 850 to $875 \mathrm{~nm}$ (inset in Fig. 3a).

Most notably, however, we observe a significant redshift $(\sim 75 \mathrm{~nm})$ of the $Q_{\mathrm{x}}$ band of $l$-P6(THS) in the F8BT matrix compared to the spectrum measured for the solution (Fig. 2a and Fig. S5 in the Supplementary Information section). In addition, as highlighted in the inset of Fig. 3a, the $Q_{\mathrm{x}}$ band is $50 \mathrm{~nm}$ narrower than in solution and exhibits some secondary components at higher energy, which are not merely the consequence of a welldefined vibronic progression. Instead, both the substantial red-shift and spectral redistribution of the oscillator strength can be attributed to a higher planarity, and reduced torsional heterogeneity of the hexamer in the solid state (either in a neat film or diluted in an F8BT matrix, Fig. S5 in the Supplementary Information section) compared to the solution, in which each porphyrin unit is free to rotate around the butadiyne links with a continuous range of torsion angles ${ }^{47}$. As previously reported $^{48}$, when the torsional degrees of freedom are constrained, for instance, when the oligomer is in a solvent with high viscosity or embedded in a solid-state a

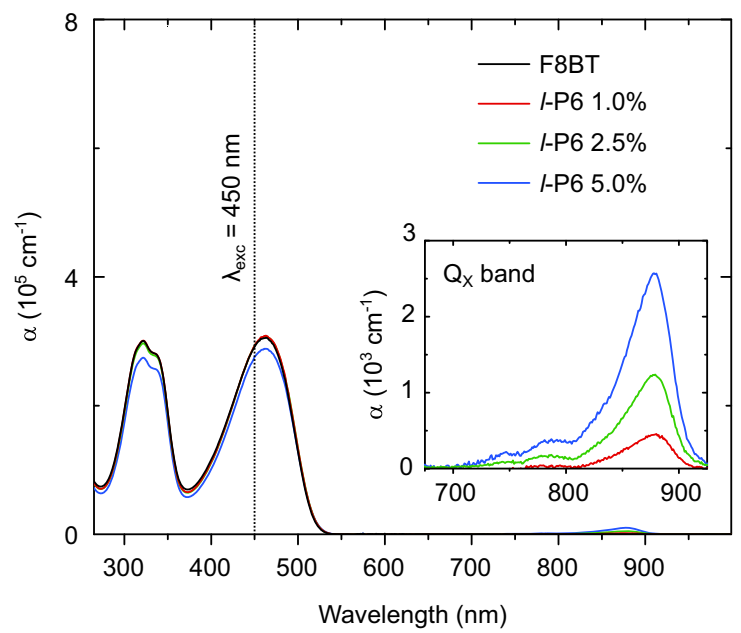

b

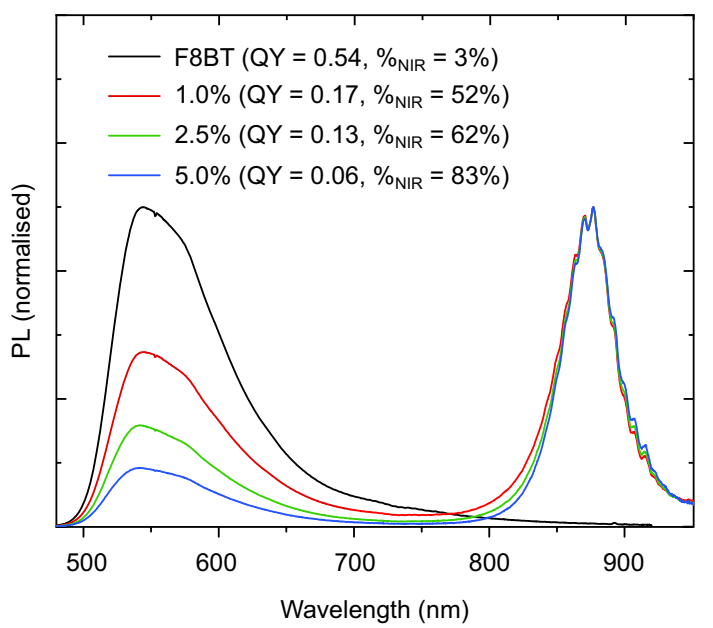

Fig. 3 Optical properties of F8BT:I-P6(THS) blends in solid-state thin films. a Absorption spectra of F8BT:I-P6(THS) blend thin films at different hexamer concentrations and $\mathbf{b}$ relative PL spectra normalized with respect to the intensity of emission at $875 \mathrm{~nm}$ (at $545 \mathrm{~nm}$ for the neat F8BT film). The inset in a highlights the evolution of the $Q_{x}$ I-P6(THS) absorption with increasing hexamer concentration. $\mathbf{b}$ The relative PLQY values (measured over the whole emission spectrum) are reported in the legend, together with the fraction of NIR emission (\% $\left.\%_{\text {NIR }}\right)$ 
matrix, planar and twisted conformers correspond to distinct spectroscopic species, each exhibiting characteristic absorption features in the $Q_{\mathrm{x}}$ manifold. Incidentally, the $Q_{\mathrm{x}}$ band profile of the blended films resembles the one we measured from a neat $l$-P6(THS) film (Fig. S5a, Supplementary Information), although in the latter case the contribution to the absorption from twisted conformers is better resolved, likely as a result of unhindered packing interactions in the absence of a solid matrix.

In the PL spectra presented in Fig. 3b, we note that emission from $\mathrm{F} 8 \mathrm{BT}$ is progressively quenched in favor of emission from $l$-P6(THS) with increasing hexamer concentration, thanks to the host-guest resonant energy transfer afforded by the spectral overlap between the host emission and the high-energy components of the hexamer Q-band. Similar to the absorption, the PL from the solidstate films is considerably red-shifted compared to that in solution and exhibits a similar scattering of the maximum value depending on the sample position investigated. We attribute this red-shift to a combination of solvation effects and planarization of the porphyrin hexamer in the solid blends. In addition, we do not observe any secondary emission at higher energy, originating from twisted conformers, as previously reported from PL measurements carried out on similar oligomers in toluene solution $^{48}$. The absence of such high-energy PL components can also be reasonably ascribed to the close packing of the hexamers in the solid state, which favors exciton funnelling towards the lowest-emitting planar conformers. Additionally, the rigidity of the oligomers in the solid-state matrix might also be responsible for the minimum Stokes' shift $(<10 \mathrm{~nm})$ on all samples, analogous to what was observed in porphyrin oligomer complexes ${ }^{39}$ and ladderlike conjugated polymers such as poly(para-phenylene $)^{51}$. However, this minimal Stokes' shift, together with the red-shift in the absorption (and PL) spectrum, may also be related to either intraoligomer, oligomer-oligomer, or polymer-oligomer dipole coupling in a J-aggregated manner ${ }^{52}$.

Concerning the PL efficiency, we summarized in the legend of Fig. 3b and in Table S1 the PLQY values of films with up to $10 \mathrm{w} / \mathrm{w} \%$ hexamer loading. Notably, these values exceed $10 \%$ in the solid state without the need for additives to limit the formation of poorly emissive aggregates $^{18}$. This achievement represents a radical advance compared to the linear P6 hexamer previously reported $^{18}$, for which the PLQY of the F8BT:P6 blend increased from $<1$ to $11 \%$ only after the addition of the metal coordinating 4-benzylpyridine.

From the different blends we tested, we note that the samples with 1 and $2.5 \mathrm{w} / \mathrm{w} \% l$-P6(THS)/polymer concentrations gave the best trade-off between high PLQY and the \% of NIR emitted photons (Fig. 3). Therefore, we focused on these two concentrations for testing as active layers in OLEDs.

\section{OLEDs}

The breakthrough above was confirmed by the fabrication and characterization of F8BT:l-P6(THS)-based OLEDs, with the device structure illustrated in Fig. 1c. The devices that gave the best NIR performance were those based on the blend with $2.5 \mathrm{w} / \mathrm{w} \% l$-P6(THS) loading. The spectra, current-voltage-radiance (JVR) and EQE versus current density characteristics are plotted in Fig. 4. The main performance parameters are summarized in Table 1.

These OLEDs were characterized by spectrally pure NIR EL up to the highest voltages tested here (Fig. 4a), in this particular case with $>98 \%$ (and more generally $>95 \%$ over the set of all devices we tested) of the photons emitted in the NIR region (at $15 \mathrm{~V}$ ) and an EL maximum at $850 \mathrm{~nm}$.
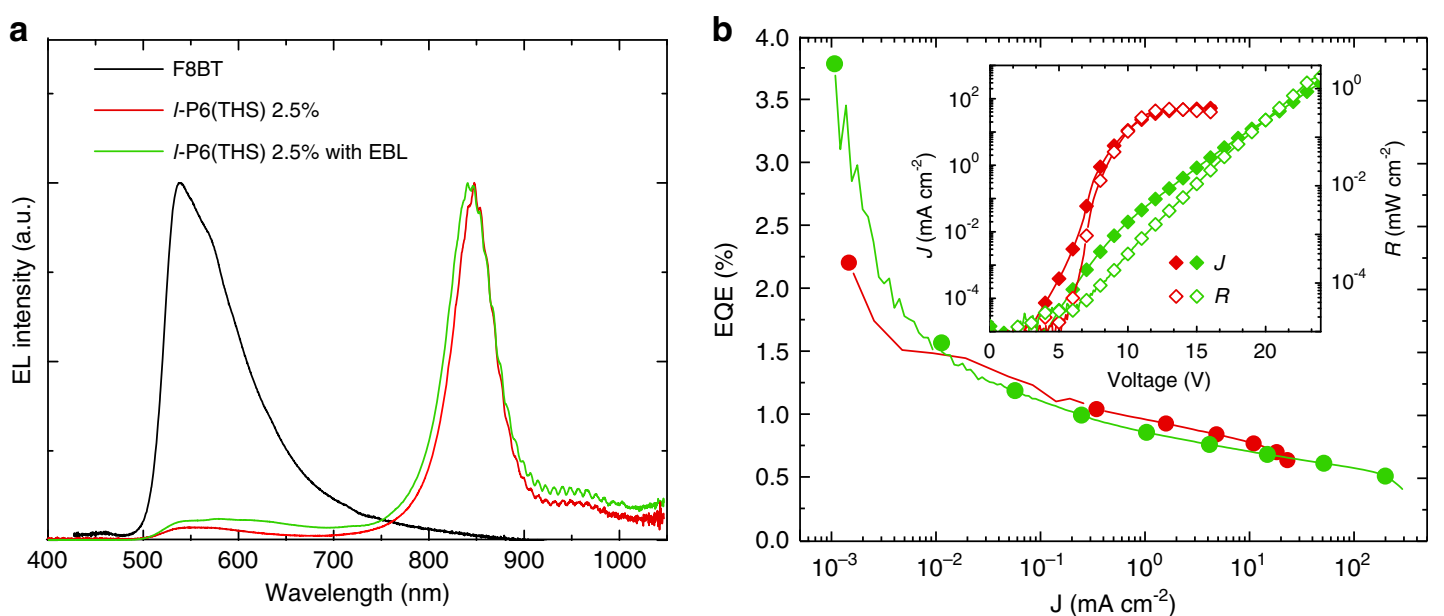

Fig. 4 OLED characteristics. EL spectra of the OLEDs incorporating F8BT:I-P6(THS) as the active layer collected at 15 and $24 \mathrm{~V}$ (i.e., the maximum radiance voltages) without and with EBL, respectively. a EQE versus current density (b), and corresponding JVR curves (inset) 
Table 1 Summary of the OLED performance parameters

\begin{tabular}{|c|c|c|c|c|c|}
\hline Sample & $V_{\mathrm{ON}}^{\mathrm{a}}[\mathrm{V}]$ & $<R_{\mathrm{MAX}}>^{\mathrm{b}}\left[\mathrm{mW} \mathrm{cm}{ }^{-2}\right]$ & $\mathrm{EQE}_{\mathrm{MAX}}{ }^{\mathrm{c}}[\%]$ & $<\mathrm{EQE}_{\mathrm{MAX}}>^{\mathrm{d}}[\%]$ & $\mathrm{EL}$ in $\mathrm{NIR}^{\mathrm{e}}[\%]$ \\
\hline F8BT:I-P6(THS) 2.5\% & $5.6 \pm 1.2$ & $0.3 \pm 0.1$ & 2.2 & $0.88 \pm 0.35$ & 98 \\
\hline F8BT:I-P6(THS) 2.5\% with EBL ${ }^{f}$ & $6.7 \pm 0.9$ & $1.9 \pm 0.6$ & 3.8 & $1.10 \pm 0.50$ & 95 \\
\hline
\end{tabular}

a Voltage at which the light output exceeds the noise level, as extrapolated from the radiance-voltage characteristics.

${ }^{\mathrm{b}}$ Average maximum radiance (for 16 devices).

'Maximum external quantum efficiency.

${ }^{d}$ Average external quantum efficiency.

ephotons emitted in the near-infrared spectral region (i.e., $\lambda>700 \mathrm{~nm}$ ).

Exciton/electron blocking layer.

Quenching of the F8BT emission in EL was significantly higher than that observed in photoluminescence (in which only $62 \%$ of the photons are emitted from the $l$-P6 (THS) dopant), thereby giving evidence for the direct formation of a significant quota of the emissive excitons on the porphyrins. EL from F8BT was also observed to increase slightly with applied voltage (see Fig. S6 in the Supplementary Information for a different diode, for which the F8BT contribution to emission is slightly higher but never higher than $5 \%$ at the highest voltage). An additional contribution to the spectral purity is a full width at half maximum (FWHM) of only $\sim 60 \mathrm{~nm}$, which is very narrow for this spectral range compared to previously reported NIR organic emitters ${ }^{6,8,10,12,14}$. Conjugated luminophores generally exhibit broad emission (and absorption) bands in the NIR region, in most cases because of conformational disorder, the charge-transfer nature of the emitting species, or both. The distinctively narrow emission band of our devices is desirable for spectroscopy (especially with a view to bio-applications and diagnostics), as it enables spectral selectivity.

Moving then to examine the performance of F8BT:l-P6 (THS) OLEDs, we observe that devices without the EBL exhibited a turn-on voltage $\left(V_{\mathrm{ON}}\right)$ of $\sim 5 \mathrm{~V}$ and a maximum radiance of $0.4 \mathrm{~mW} / \mathrm{cm}^{2}$ (Table 1). Such devices exhibited a $2.2 \%$ maximum EQE: remarkably high for OLEDs with heavy-metal-free active layers emitting at $850 \mathrm{~nm}$. Although we measured this maximum value at a current density of $0.001 \mathrm{~mA} / \mathrm{cm}^{2}$, the average maximum EQE (i.e., the average of the maximum measured over each device, for 16 diodes) was more than respectable at $0.88 \pm 0.35 \%$ and remained above $0.6 \%$ for higher current densities (up to $30 \mathrm{~mA} / \mathrm{cm}^{2}$ ).

With the two-fold aim of suppressing exciton quenching at the interface with poly(3,4-ethylene dioxythiophene) doped with poly(styrene sulfonate) (PEDOT:PSS) and improving electron-hole balance, we also fabricated diodes incorporating an additional thin $(<10 \mathrm{~nm})$ TFB exciton/electron blocking layer $(\mathrm{EBL})^{53,54}$ between the PEDOT:PSS injection layer and the emissive layer (Fig. 1c). As shown in Fig. 4b, despite the presence of a slightly larger proportion of F8BT emission (but at higher applied voltage, see Fig. 4a, vide infra) compared to the devices without the EBL, we obtained significantly higher (five times) average maximum radiances $\left(1.9 \mathrm{~mW} / \mathrm{cm}^{2}\right.$ Table 1). Most strikingly, however, we increased the average maximum EQE up to $1.1 \%$, with the best performing device yielding a maximum $\mathrm{EQE}\left(\mathrm{EQE}_{\mathrm{MAX}}\right)=$ $3.8 \%$. Although the devices were still affected by some rolloff of the efficiency with increasing current, in comparison to previously reported THS-free linear porphyrin hexamers (P6) with no benzylpyridine, the maximum EQE was two orders of magnitude higher ${ }^{18}$. We note that a "roll-off" of the efficiency with increasing current also occurred in other host-blend systems and characterized early phosphorescent OLEDs in particular but has now mostly been addressed by appropriate device and materials engineering, and we expect similar advances to be possible for the systems we present here. Therefore, the presence of such roll-off does not affect the fundamental importance of our findings regarding the general strategy to enhance luminescence in this particularly challenging spectral window.

A more detailed comparison between $l$-P6(THS) and $l$-P6(THS-free) OLEDs is reported in the Supplementary Information section (Fig. S7 and Table S4) together with results on neat F8BT control devices, that are less efficient than the NIR-emitting devices (thus ruling out that the high efficiency of the NIR OLEDs might be due to F8BT emission, please see Supplementary Information section 11 for a full discussion).

\section{A quantitative electroluminescence model}

We next consider the ratio of singlets to the total number of formed excitons $\left(r_{\mathrm{st}}\right)$, and note that the derivation of the latter on the basis of the maximum EQEs reported in Table 1 , and by using the usual expression of the IQE as the product of the electron-hole-balance factor $(\gamma)$ of $r_{\mathrm{st}}$ and of the PL efficiency $\eta$ is likely to be inaccurate, as it would ignore any triplet-to-singlet conversion process. In fact, the values derived in this way $(50 \pm 5 \%$ and $30 \pm 3 \%$ in OLEDs with and without EBL, respectively, see section 13 of the Supplementary Information section) apparently exceed the spin-statistics limit (25\%) even for conservative estimates of $\gamma(\gamma=1)$ and of the 
outcoupling factor $\left(\xi=0.75 / n^{2}\right)$, i.e., assuming all emitting chromophores oriented parallel to the device plane, with $n$ obtained via ellypsometry in our case-see Fig. S 8 in the Supplementary Information section. Notably, triplet-tosinglet conversion mechanisms such as TTA and RISC/ TADF have indeed been shown to increase the apparent $r_{\text {st }}$ over the $25 \%$ limit, not only for rubrene-doped systems ${ }^{55}$ but also for (neat) F8BT diodes in particular ${ }^{41,42}$. This is clearly relevant to our devices for which F8BT is the host.

To obtain more accurate estimates of $r_{\mathrm{st}}$, we thus developed a new quantitative model of the EQE, that not only considers singlet generation from triplets via either TTA or TADF but also, crucially, considers exciton generation directly on the guest, alongside energy transfer from the host to the guest. More specifically, in this model, the EL efficiency of the guest (porphyrin hexamer in our case) is written as the sum of two contributions: the first contribution is due to the radiative decay of singlets formed in F8BT and then transferred resonantly to $l$-P6(THS), and the second contribution is from singlets directly formed at the hexamer site. The details of such a model for the EQE of blended devices are reported in Supplementary Information section 13 and yield an $r_{\text {st }}$ of $\sim 63$ and $\sim 67 \%$ under the conservative assumptions of perfect charge balance $(\gamma=1)$ and an outcoupling efficiency $\xi \sim 0.24$ (i.e., $0.75 / \mathrm{n}^{2}$ for inplane dipole orientation $)^{42}$. In principle, if the emission could be forced to happen at an (unrealistically sharp) ideal distance from the reflective electrode $\left(\xi \sim 1.2 / n^{2} \sim 0.38\right)$, in addition to assuming an overestimated quantum yield of the porphyrin hexamers (taken to be the same as in the diluted solution, $\sim 0.31$, thus disregarding any solid-state quenching that is known to happen also in low-concentration solid solutions) and perfect electron-hole balance $(\gamma=1)$, the lower limit for $r_{\text {stP6 }}^{*}$ (defined as the "effective" singlet to total number of excitons ratio, i.e. including the contribution from singlets generated in F8BT via TTA or thermallyactivated RISC) would be 0.4 for the best performing device without EBL and 0.42 for the one with EBL. However, these assumptions would be overconservative, and we do not think they would appropriately capture the device physics (e.g., the spatial distribution of the emission region). Taken together, our results thus point to a singlet population including well over $40 \%$ of the total excitons (the value derived by Wallikewitz et al. ${ }^{41}$, as the sum of the $25 \%$ initially generated singlets and $15 \%$ additional singlets generated via a chain of consecutive TTA reactions only), thus implying a significantly greater contribution of RISC in addition to or in place of TTA.

\section{Discussion}

In conclusion, we have investigated a series of fluorescent heavy-metal-free porphyrin oligomers with increasing length to increase the mismatch of the spatial extent of singlets and triplets in order to reduce ISC, and thus mitigate the increase in the non-radiative rate $k_{\mathrm{nr}}$ while concomitantly increasing the oscillator strength (and radiative rate, $k_{\mathrm{r}}$ ). We have also combined this strategy with the molecular design of side chains to suppress aggregation quenching, thereby achieving PL efficiencies upto $30 \%$ and emission at wavelengths well above $800 \mathrm{~nm}$.

The basic photophysics and material design breakthrough has been confirmed by incorporating an F8BT:1P6(THS) blend into OLEDs, with which we demonstrated an average EQE of $1.1 \%$ and a maximum $E Q E$ of $3.8 \%$ at a peak wavelength of $850 \mathrm{~nm}$. We analysed the results within the frame of a novel quantitative model, which implies the importance of RISC/TADF to account for our EQE values beyond the apparent limit imposed by spin statistics. The EQEs presented here are, to the best of our knowledge, the highest reported so far in this spectral range from a heavy-metal-free fluorescent emitter.

Not only do our results demonstrate milder increases in $k_{\mathrm{nr}}$ with (decreasing) $E_{\mathrm{G}}$ than in the literature, but most importantly, they also provide a general strategy for designing high-luminance NIR emitters. In the short term, they may enable the further development of OLEDs in this challenging spectral range for a wide range of potential applications spanning the life sciences (biochemical wearable sensors, in vivo sub-surface bio-imaging, to name just two), security (e.g., biometrics), horticulture, and (in)visible light communications (iVLC), a serious contestant to alleviate the bandwidth demands of the imminent Internet-of-Things (IoT) revolution. Most importantly, in perspective, these findings are significant to a range of disciplines.

\section{Materials and methods \\ Materials and thin-film PL characterization}

The synthesis of the l-P6(THS) oligomer has been previously reported ${ }^{56}$. Thin films were spin-cast onto fused silica substrates from a $10 \mathrm{mg} / \mathrm{mL}$ toluene solution of the F8BT:l-P6(THS) blends. The host polymers (F8BT and TFB) were purchased from America Dye Source. The films were deposited in a $\mathrm{N}_{2}$ environment via spin-coating at $2000 \mathrm{rpm}$ for the F8BT blends and $1000 \mathrm{rpm}$ for the TFB blends to obtain a thickness of $\sim 100 \mathrm{~nm}$, measured with a Dektak profilometer. Photoluminescence was collected from an Andor Shamrock 163 spectrograph coupled with an Andor Newton EMCCD. The PLQY characterization was carried out using a $450 \mathrm{~nm}$ diode laser (Thorlabs) and an integrating sphere setup (Bentham $)^{57}$. All absorption and PL spectra were collected in air at room temperature.

\section{Transient PL characterization}

Time-resolved PL measurements were carried out in air at room temperature with a time-correlated single photon 
counting (TCSPC) spectrometer (LifeSpec Edinburgh Instruments). Samples were excited at 405 and $445 \mathrm{~nm}$ with picosecond pulsed diode lasers.

\section{OLED fabrication and characterization}

ITO substrates were cleaned in an ultrasonic bath using acetone and isopropanol, dried under a $\mathrm{N}_{2}$ stream and treated in an $\mathrm{O}_{2}$ plasma chamber for $10 \mathrm{~min}$. A $40 \mathrm{~nm}$ layer of PEDOT:PSS (purchased from Sigma Aldrich) was spin-coated at $4000 \mathrm{rpm}$ from a $2.8 \mathrm{wt} \%$ dispersion in water. The active layer was spin-coated from the same solutions used for the PL characterization, and a $\mathrm{Ca} / \mathrm{Al}$ $(30 / 200 \mathrm{~nm})$ cathode was thermally evaporated on top. For the multilayered OLEDs, a film of TFB was spin-cast onto PEDOT:PSS from a $10 \mathrm{mg} / \mathrm{ml}$ solution in toluene at $3000 \mathrm{rpm}$ and then annealed at $\sim 185^{\circ} \mathrm{C}$ for $1 \mathrm{~h}$ in a $\mathrm{N}_{2}$ environment. The TFB film was then spin-rinsed with toluene to obtain a final thickness of $<10 \mathrm{~nm}^{53}$, measured with a Dimension Icon atomic force microscope. The samples were then measured under an $\sim 10^{-2}$ mbar vacuum using a Keithley 2400 source-meter for both the current measurement and the voltage supply. The optical output of the OLEDs was measured with a calibrated silicon photodiode, and the EL spectra were collected with the same spectrometer employed for the PL experiments.

\section{Acknowledgements}

The authors gratefully acknowledge funding by EPSRC (grant EP/P006280/1, MARVEL), the European Community's Seventh Framework Programme (FP7/ 2007-2013) ITN MSCA action under Grant Agreement No. 607585 (OSNIRO), and the H2020 ETN MSCA action under grant agreement 643238 (SYNCHRONICS). E.L. gratefully acknowledges P. Mei and T. Bonfiglioli for assistance in setting up the transient electroluminescence experimental setup. F.C. acknowledges the Royal Society and the Wolfson Foundation for a Royal Society Wolfson Foundation Research Merit Award.

\section{Author details}

'Department of Physics and Astronomy and London Centre for Nanotechnology, University College London, London WC1E 6BT, UK. ${ }^{2}$ Department of Chemistry, Chemistry Research Laboratory, University of Oxford, Mansfield Road, Oxford OX1 3TA, UK. ${ }^{3}$ Dipartimento di Fisica, Università degli Studi di Pavia, Via A. Bassi, 6, Pavia 27100, Italy. ${ }^{4}$ CNR-ISMN, Istituto per lo Studio dei Materiali Nanostrutturati, Consiglio Nazionale delle Ricerche, Via P. Gobetti 101, Bologna 40129, Italy

\begin{abstract}
Author contributions
F.C. and H.L.A. conceived and supervised the work. A.M. designed the experiment, prepared the samples and carried out the measurements/data analysis (PL, EL). I.B. synthesized the porphyrin oligomers and assisted in the fabrication and characterization of the devices. A.G.R. assisted in the fabrication and characterization of the devices and carried out the AFM measurements. G.C. and M.P. carried out the characterization of the polymer film refractive indices. E.L. carried out the transient EL characterization. H.L.A. supervised the synthesis of the oligomers. F.C. supervised the photophysical and device investigations. F.C. derived the device efficiency model taking into account triplet-singlet conversion. A.M. checked and implemented the model. A.M. wrote the first draft of the manuscript, and A.M., A.G.R., H.L.A., and F.C. produced the final form of the manuscript. All authors discussed the results and contributed to the manuscript.
\end{abstract}

Conflict of interest

The authors declare that they have no conflict of interest.
Supplementary information is available for this paper at https://doi.org/ 10.1038/s41377-020-00456-8.

Received: 15 April 2020 Revised: 7 December 2020 Accepted: 15 December 2020

Published online: 21 January 2021

\section{References}

1. Kuimova, M. K. et al. Imaging intracellular viscosity of a single cell during photoinduced cell death. Nat. Chem. 1, 69-73 (2009).

2. Kim, D. Y., Lai, T.-H., Lee, J. W., Manders, J. R. \& So, F. Multi-spectral imaging with infrared sensitive organic light emitting diode. Sci. Rep. 4, 5946 (2014).

3. Tsonev, D., Videv, S. \& Haas, H. Light fidelity (Li-Fi): towards all-optical networking. SPIE Photonics West 2014-OPTO Optoelectron. Devices Mater. 9007, 900702 (2014).

4. Haigh, P. A. et al. Visible light communications: real time $10 \mathrm{Mb} / \mathrm{s}$ link with a low bandwidth polymer light-emitting diode. Opt. Express 22, 2830-2838 (2014).

5. Zampetti, A., Minotto, A. \& Cacialli, F. Near-infrared (NIR) organic light-emitting diodes (OLEDs): challenges and opportunities. Adv. Funct. Mater. 29, 1807623 (2019).

6. Tuong, Ly, K. et al. Near-infrared organic light-emitting diodes with very high external quantum efficiency and radiance. Nat. Photonics 11, 63-69 (2017).

7. Wei, Y. C. et al. Overcoming the energy gap law in near-infrared OLEDs by exciton-vibration decoupling. Nat. Photonics https://doi.org/10.1038/s41566020-0653-6 (2020).

8. Yuan, Y. et al. Over 10\% EQE near-infrared electroluminescence based on a thermally activated delayed fluorescence emitter. Adv. Funct. Mater. 27, 1700986 (2017)

9. Kim, D. H. et al. High-efficiency electroluminescence and amplified spontaneous emission from a thermally activated delayed fluorescent near-infrared emitter. Nat. Photonics 12, 98-104 (2018).

10. Tregnago, G., Steckler, T. T., Fenwick, O., Andersson, M. R. \& Cacialli, F. Thia- and selena-diazole containing polymers for near-infrared light-emitting diodes. J. Mater. Chem. C 3, 2792-2797 (2015).

11. Freeman, D. M. E. et al. Highly red-shifted NIR emission from a nove anthracene conjugated polymer backbone containing Pt(ii) porphyrins. Polym. Chem. 7, 722-730 (2016).

12. Murto, P. et al. Triazolobenzothiadiazole-based copolymers for polymer lightemitting diodes: pure near-infrared emission via optimized energy and charge transfer. Adv. Opt. Mater. 4, 2068-2076 (2016).

13. Minotto, A. et al. Efficient near-infrared electroluminescence at $840 \mathrm{~nm}$ with 'Metal-Free' small-molecule: polymer blends. Adv. Mater. 30, 1706584 (2018).

14. Zampetti, A. et al. Highly efficient solid-state near-infrared organic lightemitting diodes incorporating A-D-A dyes based on a, $\beta$-unsubstituted "BODIPY" moieties. Sci. Rep. 7, 1611 (2017).

15. Spano, F. C. \& Silva, C. H- and J-aggregate behavior in polymeric semiconductors. Annu. Rev. Phys. Chem. 65, 477-500 (2014).

16. Morgado, J. et al. Forster energy transfer and control of the luminescence in blends of an orange-emitting poly(p-phenylenevinylene) and a red-emitting tetraphenylporphyrin. J. Mater. Chem. 11, 278-283 (2001).

17. Graham, K. R. et al. Extended conjugation platinum(II) porphyrins for use in near-infrared emitting organic light emitting diodes. Chem. Mater. 23, 5305-5312 (2011)

18. Fenwick, O. et al. Linear and cyclic porphyrin hexamers as near infra-red emitters in organic light-emitting diodes. Nano Lett. 11, 2451-2456 (2011).

19. Sassi, M. et al. Near-infrared roll-off-free electroluminescence from highly stable diketopyrrolopyrrole light emitting diodes. Sci. Rep. 6, 34096 (2016).

20. McQuade, D. T., Kim, J. \& Swager, T. M. Two-dimensional conjugated polymer assemblies: interchain spacing for control of photophysics. J. Am. Chem. Soc. 122, 5885-5886 (2000).

21. Cacialli, F. et al. Cyclodextrin-threaded conjugated polyrotaxanes as insulated molecular wires with reduced interstrand interactions. Nat. Mater. 1, 160-164 (2002).

22. Brovelli, S. et al. White electroluminescence by supramolecular control of energy transfer in blends of organic-soluble encapsulated polyfluorenes. Adv. Funct. Mater. 20, 272-280 (2010).

23. Brovelli, S. et al. Emission color trajectory and white electroluminescence through supramolecular control of energy transfer and exciplex formation in 
binary blends of conjugated polyrotaxanes. Adv. Funct. Mater. 22, 4284-4291 (2012).

24. Englman, R. \& Jortner, J. The energy gap law for radiationless transitions in large molecules. Mol. Phys. 18, 145-164 (1970).

25. Thomas, T. H. et al. Short contacts between chains enhancing luminescence quantum yields and carrier mobilities in conjugated copolymers. Nat. Commun. 10, 2614 (2019).

26. Zhao, B. et al. High-efficiency perovskite-polymer bulk heterostructure lightemitting diodes. Nat. Photonics 12, 1-10 (2018).

27. Supran, G. J. et al. High-performance shortwave-infrared light-emitting devices using core-shell (PbS-CdS) colloidal quantum dots. Adv. Mater. 27, 1437-1442 (2015).

28. Wang, N. et al. Perovskite light-emitting diodes based on solution-processed self-organized multiple quantum wells. Nat. Photonics 10, 699-704 (2016).

29. Xiao, Z. et al. Efficient perovskite light-emitting diodes featuring nanometresized crystallites. Nat. Photonics 11, 108-115 (2017).

30. Yuan, Y. et al. Boosting efficiency of near-infrared organic light-emitting diodes with Os(II)-based pyrazinyl azolate emitters. Adv. Funct. Mater. 30, 1906738 (2020).

31. Yang, X. et al. Enhancing molecular aggregations by intermolecular hydrogen bonds to develop phosphorescent emitters for high-performance near-infrared OLEDs. Adv. Sci. 6, 1801930 (2019).

32. Chen, Z. et al. A simple and efficient approach toward deep-red to nearinfrared-emitting iridium(iii) complexes for organic light-emitting diodes with external quantum efficiencies of over 10\%. Chem. Sci. 11, 2342-2349 (2020).

33. Congrave, D. G. et al. A simple molecular design strategy for delayed fluorescence toward $1000 \mathrm{~nm}$. J. Am. Chem. Soc. 141, 18390-18394 (2019).

34. Xue, J. et al. Highly efficient thermally activated delayed fluorescence via Jaggregates with strong intermolecular charge transfer. Adv. Mater. 31, 1808242 (2019)

35. Ostrowski, J. C., Susumu, K., Robinson, M. R., Therien, M. J. \& Bazan, G. C. Nearinfrared electroluminescent light-emitting devices based on ethyne-bridged porphyrin fluorophores. Adv. Mater. 15, 1296-1300 (2003).

36. Duncan, T. V., Ishizuka, T. \& Therien, M. J. Molecular engineering of intensely near-infrared absorbing excited states in highly conjugated oligo(porphinato) zinc-(polypyridyl)metal(II) supermolecules. J. Am. Chem. Soc. 129, 9691-9703 (2007).

37. Duncan, T. V., Frail, P. R., Miloradovic, I. R. \& Therien, M. J. Excitation of highly conjugated (Porphinato)palladium(II) and (Porphinato)platinum(II) oligomers produces long-lived, triplet states at unit quantum yield that absorb strongly over broad spectral domains of the NIRT. J. Phys. Chem. B 114, 14696-14702 (2010).

38. Anderson, H. L. Building molecular wires from the colours of life: conjugated porphyrin oligomers. Chem. Commun. 0, 2323-2330 (1999).

39. Chang, M. H., Hoffmann, M., Anderson, H. L. \& Herz, L. M. Dynamics of excitedstate conformational relaxation and electronic delocalization in conjugated porphyrin oligomers. J. Am. Chem. Soc. 130, 10171-10178 (2008).

40. Yong, C.-K. et al. Ultrafast delocalization of excitation in synthetic lightharvesting nanorings. Chem. Sci. 6, 181-189 (2015).
41. Wallikewitz, B. H., Kabra, D., Gélinas, S. \& Friend, R. H. Triplet dynamics in fluorescent polymer light-emitting diodes. Phys. Rev. B 85, 22-25 (2012).

42. Dey, A., Rao, A. \& Kabra, D. A complete quantitative analysis of spatio-temporal dynamics of excitons in functional organic light-emitting diodes. Adv. Opt. Mater. 5, 1600678 (2017)

43. Caspar, J. V., Kober, E. M., Sullivan, B. P. \& Meyer, T. J. Application of the energy gap law to the decay of charge-transfer excited states. J. Am. Chem. Soc. 104, 630-632 (1982).

44. Caspar, J. V. \& Meyer, T. J. Application of the energy gap law to nonradiative, excited-state decay. J. Phys. Chem. 87, 952-957 (1983).

45. Wilson, J. S. et al. The energy gap law for triplet states in pt-containing conjugated polymers and monomers. J. Am. Chem. Soc. 123, 9412-9417 (2001).

46. Duncan, T. V., Susumu, K, Sinks, L. E. \& Therien, M. J. Exceptional near-infrared fluorescence quantum yields and excited-state absorptivity of highly conjugated porphyrin arrays. J. Am. Chem. Soc. 128, 9000-9001 (2006).

47. Peeks, M. D., Neuhaus, P. \& Anderson, H. L. Experimental and computational evaluation of the barrier to torsional rotation in a butadiyne-linked porphyrin dimer. Phys. Chem. Chem. Phys. 18, 5264-5274 (2016).

48. Winters, M. U. et al. Photophysics of a butadiyne-linked porphyrin dimer: influence of conformational flexibility in the ground and first singlet excited state. J. Phys. Chem. C 111, 7192-7199 (2007).

49. Beljonne, D., Shuai, Z., Pourtois, G. \& Bredas, J. L. Spin-orbit coupling and intersystem crossing in conjugated polymers: a configuration interaction description. J. Phys. Chem. A 105, 3899-3907 (2001).

50. Kuimova, M. K. et al. Determination of the triplet state energies of a series of conjugated porphyrin oligomers. Photochem. Photobiol. Sci. 6, 675-682 (2007).

51. Grüner, J. et al. Electroluminescence and photoluminescence investigations of the yellow emission of devices based on ladder-type oligo(para-phenylene)s. Synth. Met. 67, 181-185 (1994).

52. Kärnbratt, J., Gilbert, M., Sprafke, J. K., Anderson, H. L. \& Albinsson, B. Selfassembly of linear porphyrin oligomers into well-defined aggregates. J. Phys. Chem. C 116, 19630-19635 (2012).

53. Kim, J. S., Friend, R. H., Grizzi, I. \& Burroughes, J. H. Spin-cast thin semiconducting polymer interlayer for improving device efficiency of polymer light-emitting diodes. Appl. Phys. Lett. 87, 80-83 (2005).

54. Lazzerini, G. M. et al. Low-temperature treatment of semiconducting interlayers for high-efficiency light-emitting diodes based on a green-emitting polyfluorene derivative. Appl. Phys. Lett. 99, 2-4 (2011).

55. Kondakov, D. Y., Pawlik, T. D., Hatwar, T. K. \& Spindler, J. P. Triplet annihilation exceeding spin statistical limit in highly efficient fluorescent organic lightemitting diodes. J. Appl. Phys. 106, 124510 (2009).

56. Tait, C. E. et al. Reveals triplet state delocalization in a series of cyclic and linear p-conjugated porphyrin oligomers. J. Am. Chem. Soc. 137, 8284-8293 (2015).

57. de Mello, J. C., Wittmannn, H. F. \& Friend, R. H. An improved experimental determination of external photoluminescence quantum efficiency. Adv. Mater. 9, 230 (1997)

58. Chua, L. et al. General observation of n-type field-effect behaviour in organic semiconductors. Nature 434, 194-199 (2005). 